\section{ONOMÁVAEIN}

Revista semestral de lingüística, filología y traducción
PONTIFICIA UNIVERSIDAD CATÓLICA DE CHILE CATOLICA DE CHILE
FACULTAD DE LETRAS

\title{
El desarrollo de la escritura argumentativa académica: los marcadores discursivos ${ }^{1}$
}

The development of academic argumentative writing: discourse markers

\section{María Constanza Errázuriz Cruz}

Pontificia Universidad Católica de Chile Chile 


\section{Resumen}

Las dificultades de los estudiantes universitarios y de programas de formación inicial docente para producir textos en ámbitos académicos han sido evidenciadas por la prueba INICIA (evaluación de los egresados de pedagogía) y diversas investigaciones del área de la alfabetización académica (Carlino, 2005; Castelló, 2009; Foster y Russell, 2002; Parodi, 2010; Swales y Feak, 2004). Es por esta razón que en este estudio se abordaron los problemas de escritura que actualmente tienen los estudiantes, a partir del análisis del uso de los marcadores discursivos en sus textos argumentativos (Ducrot, 2001; Martín Zorraquino y Portolés, 1999; Montolío, 2001; Portolés, 1998 y 2010). El corpus escogido consistió en 40 ensayos escritos por estudiantes de Pedagogía General Básica y 40 textos del grupo anterior luego de la aplicación de un plan de alfabetización académica externo a la investigación. Con respecto a la metodología, corresponde a un estudio descriptivo y cuantitativo de los ensayos y sus conectores, pues se evaluó la efectividad de los ensayos mediante una rúbrica y se analizó su estructura y el funcionamiento de sus nexos. De este modo, se comprobó el rol preponderante que poseen los marcadores discursivos para el procesamiento de las inferencias y la producción de textos coherentes, ya que se observó la correspondencia que existe entre la adecuación de estos nexos y la progresión lógica de los ensayos en donde funcionan. Por último, también se constató que el desarrollo de la escritura académica a través de un plan dirigido a sus docentes tuvo un impacto positivo en las producciones de textos de los estudiantes.

Palabras clave: marcador discursivo; alfabetización académica; texto argumentativo; ensayo.

\section{Abstract}

The difficulties that college students and initial teacher training programs have to produce academic texts have been highlighted by INICIA test (assessment of graduates of education) and several studies in the area of academic literacy (Carlino, 2005; Castelló, 2009; Foster \& Russell, 2002; Parodi, 2010; Swales \& Feak, 2004). It is for this reason that this study addressed problems of writing that students have nowadays, through the analysis of the use of discourse markers in argumentative texts (Ducrot, 2001; Martín Zorraquino \& Portolés, 1999; Montolío, 2001; Portolés, 1998 \& 2010). The corpus consisted of 40 essays written by students of Elementary Education and 40 essays of the previous group after an academic literacy plan.
With regard to the methodology, it is a descriptive and quantitative study of texts and discourse markers, as they evaluated the effectiveness of essays using a rubric and analyzed its structure and functioning of their discourse markers. The correspondence between the appropriateness of these links and the logical progression of essays in which they operate was observed, and thus it was found that discourse markers have a prominent role in inference processing and producing coherent texts. Finally, it was also found that the development of academic writing through a plan aimed to their teachers had a positive impact on the students' texts.

Keywords: discourse marker; academic literacy; argumentative texts; essays.

1 Agradecimientos: Parte de este estudio fue financiado por Conicyt, Fondecyt Iniciación N 11130560 "Tipos de teorías implícitas sobre los procesos de escritura de estudiantes, formadores y profesores tutores de programas de formación inicial docente: Análisis de una relación significativa para el desarrollo de las competencias de escritura”.

Esta investigación corresponde a un avance de la planteada en Errázuriz (2012). 


\section{Introducción}

El interés por indagar en el comportamiento de los marcadores discursivos en la escritura de textos argumentativos, en situaciones académicas, surge de la comprobación de que estos cumplen un rol clave en la orientación de las inferencias y en el procesamiento de la información en la lectura y, especialmente, en el proceso de producción de textos (Fuentes, 1996; Martín Zorraquino y Portolés, 1999; Montolío, 2001; Portolés, 1998 y 2010). Además, junto con el expositivo, el discurso argumentativo es muy frecuente en el ámbito académico, pues los estudiantes, desde el inicio de sus vidas universitarias, deben fundamentar sus opiniones y comprender el discurso razonado de otros (Brockriede, 1993; Castro, Hernández y Sánchez, 2010; Toulmin, 1993; Vignaux, 1986). Para ello, necesitan manejar las técnicas básicas de la construcción del ensayo argumentativo y académico breve (Castro y otros, 2010; Weinberg, 2007), según las exigencias básicas de evaluaciones chilenas como el Examen de Comunicación Escrita UC y la prueba INICIA, cuyas concepciones de este género discursivo coinciden con la tradición anglosajona de cinco párrafos: uno para la introducción y la tesis, tres para cada argumento y el contraargumento, y el último para las conclusiones. En el aprendizaje de estos movimientos retóricos, el manejo de los marcadores desempeña un papel importante, ya que permite organizar y guiar las implicaturas, dotando al texto de coherencia (Ducrot, 2001; Grice, 1975; Sperber y Wilson, 1994).

Por otra parte, actualmente los estudiantes de educación superior presentan problemas para producir textos adecuados al contexto académico (Carlino, 2005; Castelló, 2009; Parodi, 2010). Esto parece aún más preocupante en los programas de formación inicial docente chilenos, cuya calidad ha sido cuestionada (Oliva y otros, 2010; Meckes, 2011; Mineduc, 2005). Así lo corroboran los resultados de la última prueba INICIA², instrumento que evalúa la redacción de un ensayo entre diversas competencias en los egresados de carreras de educación, cuyos resultados señalan que solo el $37 \%$ de los egresados logró un nivel adecuado y el 63\% no alcanzó este nivel. También existe una evaluación de comunicación escrita realizada por la Pontificia Universidad Católica de Chile que se aplica a todos los estudiantes que ingresan a estudiar y consiste en evaluar la redacción de un ensayo a través de una rúbrica, cuyos resultados se organizan en una escala del 1 a 5. El promedio de los puntajes de los estudiantes de Pedagogía Básica en los dos últimos años es de un 2,5, vale decir, poseen menos de un 50\% de dominio, pues el puntaje de aprobación es 3 y, además, están bajo el promedio de la universidad.

En este sentido, si bien anteriormente la composición de un escrito era considerada como un producto y una simple encodificación o una transcripción de la oralidad (Errázuriz, 2010; Ferreiro, 2008; Martínez, 2001), en la actualidad se sabe que la situación de comunicación del discurso escrito es totalmente distinta a la del discurso oral, pues no están presentes simultáneamente el escritor y su lector, por lo que la tarea de escribir es llamada "producción de texto", debido a que es considerada como un proceso en donde cada momento es tan importante como el producto final (Álvarez y Ramírez, 2006; Bereiter y Scardamalia, 1987; Camps, 2003; Cassany, 2000; Didactext, 2003; Flower y Hayes, 1981). Sin embargo, tal parece que este enfoque no se aplica habitualmente en clases al momento de producir textos académicos (Carlino, 2005, 2009; Carlino y Padilla, 2010; Castelló, 2009; Errázuriz y Fuentes, 2012).

En consecuencia, este trabajo precisamente pretende explorar cuál es el uso real de los marcadores discursivos en los ensayos argu-

2 Esta evaluación mide los conocimientos disciplinares y pedagógicos y las competencias comunicativas y tecnológicas de estudiantes egresados de pedagogía, para constatar la calidad de los programas de formación. 
mentativos escritos por estudiantes del área de educación en situaciones académicas, antes y después de la aplicación de un plan de escritura académica. Por lo tanto, tiene por objetivo general analizar los ensayos escritos por estudiantes de Educación General Básica y sus marcadores discursivos, antes y después de un plan de alfabetización académica. De este modo, se espera comprobar que la calidad y la congruencia de un texto son resultados que, en parte, se deben a una adecuada utilización de los marcadores discursivos en textos argumentativos académicos durante el proceso de redacción. Es decir, un uso lógico y pertinente de los conectores debería tener, en general, como consecuencia un texto coherente y apropiado.

Por último, respecto de la estructura de este artículo, está compuesto, en primer lugar, por la fundamentación teórica, que presenta los conceptos, modelos y perspectivas más relevantes para el análisis de los marcadores en la producción de ensayos argumentativos; luego, le sigue el diseño metodológico, que presenta la descripción del corpus, los instrumentos y los métodos utilizados en el análisis; finalmente, se presentan los resultados y las conclusiones.

\section{Fundamentación teórica}

\subsection{El texto argumentativo}

Determinar en qué consiste la argumentación no es una tarea fácil, ya que existen múltiples definiciones como "lo compuesto por argumentos", "serie de argumentos que tienden a una conclusión", "conjunto de razonamientos que tienden a una tesis", "arte de argumentar" (Vignaux, 1986: 14). Todas ellas resultan muy vagas y varían de acuerdo con el aspecto que se adopte. De hecho, hay numerosos puntos de vista para abordar la argumentación, pero en esta investigación se consideraron algunos de ellos.

En este sentido, según Brockriede (1993), el argumento no es algo externo o concreto, sino un punto de vista que toma y percibe cada persona. De este modo, es cambiante y subjetivo, pues puede estar en cualquier lugar. Por lo tanto, el argumento cae en el terreno de lo problemático, ya que genera una discusión que no es fácil de resolver, pues no depende de una lógica categórica del sí o no, sino que depende de una lógica variable del continuo "más o menos".

Con respecto al texto argumentativo, tradicionalmente se ha considerado que está compuesto básicamente de una tesis, premisas compartidas culturalmente, un cuerpo argumentativo y una conclusión (Anscombre y Ducrot, 1994; Ducrot, 2001). Al interior de este esquema, la tesis constituye el núcleo y debe presentársela claramente aunque puede quedar implícita, para luego exponer los argumentos que la apoyan, y así llegar a una conclusión para confirmarla o refutarla. Sin embargo, esta estructura resulta demasiado simple, pues elide o invisibiliza ciertas partes y pasos del proceso argumentativo. Otras perspectivas, como las de Toulmin (1958 y 1993), Perelman y Olbrechts Tyteca (2009) y Van Eemeren y Grootendorst (2002), consideran la argumentación como un producto, una defensa jurídica o un proceso comunicativo oral, respectivamente, pero no abordan la argumentación como texto y género discursivo ni su proceso de escritura.

Es por esto que en esta investigación se utilizó el esquema argumentativo global (Errázuriz, 2010 y 2012), el cual comprende de un modo más amplio los componentes de la argumentación escrita y del ensayo y el proceso de producción escrita. Este modelo se basa en la estructura tradicional del texto argumentativo, constituida por una tesis, un cuerpo argumentativo y una conclusión (Ducrot, 2001; Lo Cascio, 1998; Toulmin, 1958 y 1993; Weston, 1994; Van Dijk, 1997; Wenzel, 1993), y a partir de la extensión de esta se desarrolló un esquema (figura 1) que permitiera integrar las distintas manifestaciones de la argumentación. En primer lugar, el marco inicial -que corresponde a la introducción y exhibe la controversia - puede estar conformado por elementos que son opcionales, como la presen- 
tación del tema, los objetivos o la descripción del problema. En segundo lugar, el desarrollo del tema, el cual corresponde al cuerpo de la argumentación y muestra las posiciones a favor y en contra, puede estar integrado por uno o más argumentos conectados con uno o más contraargumentos. Por último, en el marco final -que corresponde a la conclusión y clausura la argumentación- pueden encontrarse componentes que son opcionales, como el cierre del tema, la síntesis, la discusión, las proyecciones y la recapitulación. Ahora bien, en cualquiera de estas tres etapas se puede incluir o introducir la tesis u opinión sobre el tópico tratado. Por tanto, la tesis puede presentarse tanto en el marco inicial como en el desarrollo del tema y en el marco final, dependiendo de la estructura argumentativa escogida y de la estrategia que se esté utilizando.

Con respecto al género discursivo ensayo argumentativo, es uno de los textos de mayor circulación en las humanidades (Castro y otros, 2010; Errázuriz y Fuentes, 2012; Errázuriz y Sánchez, enviado; Parodi, 2012; Weinberg, 2007), por el nivel de dificultad que ofrece al abordar la argumentación. Además, Anscombre y Ducrot
(1994) señalan que todo texto tiene un propósito final argumentativo, aunque este sea subyacente. Asimismo, es el género utilizado para desarrollar la escritura académica en diversos planes de alfabetización académica en el mundo anglosajón (Bazerman y otros, 2005; Foster y Russell, 2002; Slotte y Lonka, 2001), pues constituye un primer paso para la elaboración de artículos científicos (Sabaj y Landea, 2012) y es evaluado por varias universidades chilenas y en la prueba INICIA. En consecuencia, como es uno de los géneros académicos más leídos y escritos por los estudiantes universitarios chilenos de carreras de pedagogía, resulta interesante analizar su estructura y la utilización de sus marcadores discursivos, para corroborar su efectividad.

\subsection{Los marcadores discursivos}

Con respecto a los marcadores discursivos, la definición operacional que se utilizó en esta investigación es de Portolés (1998) y considera estas unidades lingüísticas como

unidades lingüísticas invariables, no ejercen una función sintáctica en el marco de la predicación oracional y poseen un cometido coincidente en el discurso: el de guiar, de acuerdo con sus distin-

\section{FIGURA 1}

Esquema del texto argumentativo

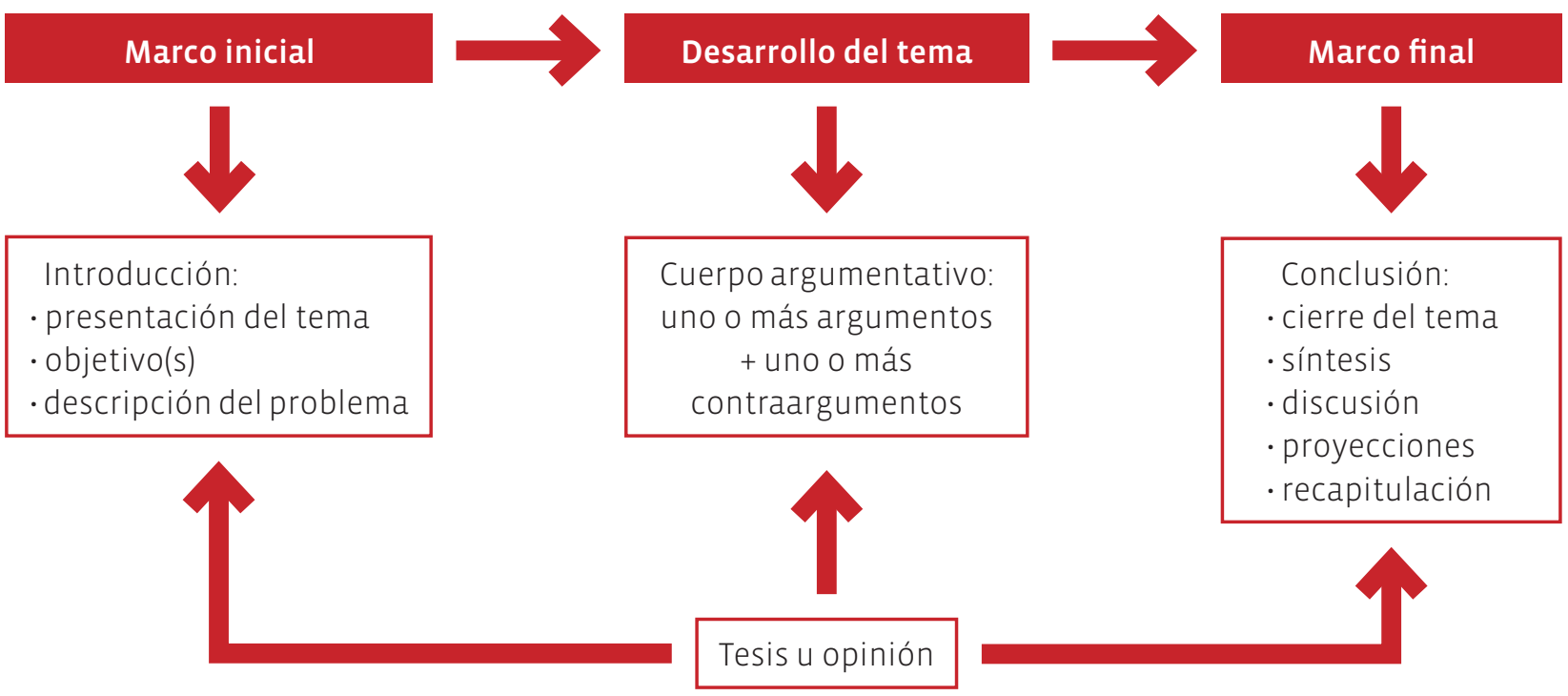


tas propiedades morfosintácticas, semánticas y pragmáticas, las inferencias que se realizan en la comunicación (1998: 25-26).

Siguiendo esta definición, es necesario precisar que el término marcador discursivo se presenta como el más adecuado para denominar a estas unidades, pues abarca las diversas funciones que son capaces de desempeñar. En efecto, los marcadores no solo enlazan los diferentes sintagmas de un texto, sino que también son los encargados de relacionar las intenciones y supuestos que se esconden tras los enunciados de un discurso (Loureda y Acín, 2010; Montolío, 2001; Portolés, 1998 y 2010).

En este sentido, los marcadores se encuentran en el límite oracional y su función es organizar el texto dotándolo de coherencia y así guiar la dirección del pensamiento del hablante para que la información proporcionada por el texto sea clara y ordenada. Asimismo, según la perspectiva de Van Dijk (1983), los marcadores son los elementos encargados de organizar la información contenida en el texto; en consecuencia, forman parte de la macroestructura.

En esta investigación también se abordaron distintas perspectivas sobre las funciones del marcador discursivo, como implicaturas que relevan información implícita o las conclusiones, según Grice (1975); presuposiciones que explicitan una serie de creencias, de acuerdo con Ducrot (2001); orientadores de inferencias y de suposiciones contextuales, siguiendo a Sperber y Wilson (1994) y Blakemore (2002), e instrucciones argumentativas que indican la fuerza argumentativa de los enunciados, según Anscombre (1995) y Anscombre y Ducrot (1994).

Para la identificación precisa de estos elementos que enlazan los enunciados y guían el procesamiento de la información y de las inferencias en relación al contexto del discurso proporcionándole sentido, fue necesario profundizar en sus propiedades gramaticales. Según Fuentes (1996), Martín Zorraquino y Portolés (1999) y Portolés (2010), estas son:
1. No tienen función sintáctica ni referencial

2. Se ubican en el margen oracional (Silva-Corvalán, 2001: 218).

3. Son invariables.

4. Pueden variar su posición (inicial o intermedia).

5. Pueden coordinarse entre sí.

6. No tienen determinantes y no se combinan con otros elementos (sin negación, ni graduación).

7. Poseen significado de unión y procesamiento.

La clasificación de los marcadores discursivos que se utilizará (figura 2) ha sido elaborada a partir de las tipologías revisadas de Calsamiglia y Tusón (2001), Cueva (2008), Fuentes (1996), Lo Cascio (1991), Martín Zorraquino y Portolés (1999), Pons (1998), Portolés (1998). Se centra en el plano enunciativo, el cual se focaliza en el desarrollo y construcción de la comunicación por parte del hablante. A partir del punto de vista expuesto y según los planteamientos de Pottier (1993) (cfr. Pérez y Vega, 2001), es posible considerar el proceso de construcción del discurso como un continuo compuesto por un inicio (visión prospectiva), una continuación (visión coincidente) y un cierre (visión retrospectiva). Así, los marcadores discursivos se clasificarán de acuerdo con su valor en los tres siguientes grupos (Errázuriz, 2012): los marcadores con valor de inicio, que corresponden a enlaces con valor de topicalización (introducen un tópico); los marcadores con valor de continuación, que corresponden a los enlaces de adición, continuidad, orden (enumeración y distribución), conformidad (equivalencia, inclusión, causalidad) y disconformidad (oposición), y los marcadores con valor de cierre, que usualmente presentan una perspectiva de epílogo, conclusión o resumen.

\subsection{La producción de textos}

Una de las aproximaciones más relevantes que se consideró en este trabajo para el análisis y evaluación de los ensayos es la propuesta de Bereiter y Scardamalia (1987) sobre decir y transformar el conocimiento. Este enfoque señala la importancia que tiene para el escritor ser capaz 


\section{FIGURA 2}

Clasificación de los marcadores discursivos

\begin{tabular}{|c|c|c|c|c|c|c|}
\hline INICIO & \multicolumn{5}{|c|}{ CONTINUACIÓN } & CIERRE \\
\hline topicalizadores & adición & continuidad & orden & conformidad & disconformidad & \\
\hline $\begin{array}{l}\text { a propósito de, } \\
\text { con respecto a, } \\
\text { en cuanto a, en } \\
\text { lo concerniente } \\
\text { a, en lo que } \\
\text { atañe a, en } \\
\text { relación con, } \\
\text { por lo que se } \\
\text { refiere a, por lo } \\
\text { que respecta } \\
\text { a, respecto } \\
\text { de, según, a } \\
\text { mi juicio, en } \\
\text { mi opinión... }\end{array}$ & $\begin{array}{l}\text { además, } \\
\text { asimismo, } \\
\text { encima, } \\
\text { incluso, } \\
\text { todavía, } \\
\text { más/menos, } \\
\text { igualmente, } \\
\text { ante todo, de } \\
\text { igual modo, } \\
\text { también, } \\
\text { tampoco, en } \\
\text { ese sentido... }\end{array}$ & $\begin{array}{c}\text { bueno, claro, } \\
\text { desde luego, } \\
\text { por supuesto, } \\
\text { por otrolado, } \\
\text { también, } \\
\text { ciertamente, } \\
\text { por cierto, } \\
\text { probablemente, } \\
\text { sin duda, } \\
\text { definitivamente, } \\
\text { en general... }\end{array}$ & $\begin{array}{c}\text { enumeración: } \\
\text { primero, } \\
\text { segundo, } \\
\text { tercero, en } \\
\text { primerlugar, } \\
\text { en segundo } \\
\text { lugar... } \\
\text { distribución: } \\
\text { a } \\
\text { continuación, } \\
\text { primero, } \\
\text { luego, } \\
\text { después, } \\
\text { finalmente, } \\
\text { por último, } \\
\text { por un lado, } \\
\text { porotro, por } \\
\text { una parte, } \\
\text { por otra... }\end{array}$ & $\begin{array}{c}\text { equivalencia: } \\
\text { es decir, o } \\
\text { sea, vale } \\
\text { decir, quiero } \\
\text { decir, en otras } \\
\text { palabras, } \\
\text { mejor dicho, } \\
\text { en el fondo... } \\
\text { inclusión: } \\
\text { así, por } \\
\text { ejemplo... } \\
\text { causalidad: } \\
\text { de este modo, } \\
\text { entonces, en } \\
\text { efecto, en } \\
\text { consecuencia, } \\
\text { así, por } \\
\text { consiguiente, } \\
\text { por ende, } \\
\text { por lo tanto, } \\
\text { de hecho, } \\
\text { de manera, } \\
\text { por cierto... }\end{array}$ & $\begin{array}{c}\text { oposición: } \\
\text { de todos } \\
\text { modos, de } \\
\text { cualquier modo, } \\
\text { en cambio, } \\
\text { en todo caso, } \\
\text { no obstante, } \\
\text { pero, por el } \\
\text { contrario, sin } \\
\text { embargo, ahora } \\
\text { bien, apesar de } \\
\text { todo, después } \\
\text { de todo, } \\
\text { antes bien... }\end{array}$ & $\begin{array}{l}\frac{\text { de epílogo o }}{\text { recapitulación: }} \\
\text { al fin yal cabo, } \\
\text { en conclusión, } \\
\text { en definitiva, } \\
\text { en fin, en } \\
\text { resumen, } \\
\text { en síntesis, } \\
\text { en suma, } \\
\text { finalmente, } \\
\text { para concluir, } \\
\text { por último, } \\
\text { para terminar... }\end{array}$ \\
\hline
\end{tabular}

de transformar el conocimiento que se investiga y no solo reproducirlo. Esto es apoyado por Ruiz (2009), quien señala que la enseñanza de la lectura y la escritura ha estado determinada por la dependencia de lo literal, es decir, por la reproducción del conocimiento, por lo que el gran desafío en el desarrollo de estas competencias está en el aprendizaje de habilidades para construir y transformar el conocimiento desde una perspectiva crítica y creativa, lo que es indispensable en la elaboración de ensayos.

En relación con estrategias de composición, se siguió en parte la propuesta de Flower y Hayes (1981). Según esta, la escritura es un proceso compuesto de momentos recursivos que se pueden imbricar, superponer y repetir. Por tanto, requiere una reflexión de parte del escritor en cuanto a sus conocimientos, el texto, el tema, el léxico y el registro, pues el escritor debe seleccionar el destinatario, el propósito comunicativo, el tipo de texto y los modos de enunciación, entre otros. Este proceso tiene tres componentes: la memoria de largo plazo, el ambiente de trabajo y la escritura que posee los momentos de planificación, traducción y revisión (Flower, 1989).

Sin embargo, el modelo anterior no considera la dimensión sociocultural de la escritura y se centra excesivamente en los aspectos cognitivos (Álvarez y Ramírez, 2006); es por eso que también se abordó el modelo sociocognitivo, pragmalingüístico y didáctico del grupo Didactext (2003). Este enfoque plantea que la producción de textos es un macroproceso en el que interactúan tres ámbitos: el cultural, que comprende las prácticas humanas en la redacción de textos y sus géneros discursivos (Bajtin, 1982; Bronckart, 2010); el contextual, que se refiere a los contextos de producción y la audiencia (Nystrand, 1989), y el individual, que alude a los recursos y estrategias del escritor. Estos ámbitos se articulan en cuatro 
fases: acceso al conocimiento, planificación, producción y revisión

Además, se incluyeron elementos del modelo de Candlin y Hyland (1999), que desarrolla la escritura como texto (Beaugrande y Dressler, 1997), proceso y práctica social, especialmente en contextos educativos y profesionales, en donde circulan diversos géneros que deben ser leídos para ser escritos adecuadamente (Bajtin, 1982; Bronckart, 2010; Parodi y otros, 2010, y Teberosky, 2009). Por último, también se consideraron aspectos del modelo de Grabe y Kaplan (1996), que define la escritura como una actividad humana inserta en la cultura: por eso se centra en las prácticas culturales y sus aspectos etnográficos. Como metodología propone las preguntas quién escribe qué, a quién, con qué propósito, por qué y cómo.

Estos enfoques generan un desafío para una evaluación más auténtica y formativa tanto en la escuela como en la universidad, pues es necesario considerar no solo el texto final, sino también las planificaciones y avances del proceso (Ahumada, 2005; Condemarín y Medina, 2000; OREALC/UNESCO, LLECE y Atorresi, 2010, y Tragant, 1996).

Por último, también se consideró el enfoque de la alfabetización académica (AA) que aborda la escritura como una potente herramienta de aprendizaje y construcción de conocimientos que permite generar mayores aprendizajes en las disciplinas insertas en contextos académicos (Bazerman y otros, 2005; Carlino, 2005 y 2013; Castelló, 2009; Foster y Russell, 2002; García Parejo, 2011; Miras y Solé, 2009; Swales y Feak, 2004; Teberosky, 2009). En este sentido, según estos planteamientos se considera que es labor de las universidades introducir a los alumnos en el quehacer académico, es decir, en el análisis y escritura de textos en cada disciplina a lo largo del currículo, pues solo los expertos de cada área saben cómo comprender y producir textos en su ámbito. Esto es aún más evidente en la formación inicial docente (FID), pues el aprendizaje está mediado por el lenguaje, que funciona como una herramienta epistémica.

\section{Diseño metodológico}

Esta investigación se enmarca como un estudio descriptivo cuantitativo -mediante el programa estadístico SPSS- de los ensayos escritos por los alumnos y el funcionamiento de sus marcadores discursivos antes y después de la aplicación externa a la investigación de un plan de alfabetización académica que duró ocho meses y que involucró la capacitación y participación de los académicos formadores de estos estudiantes. Las hipótesis eran: 1) Existe relación entre la efectividad de los ensayos escritos por los estudiantes de la muestra y la adecuación del uso de sus marcadores. 2) Es posible que un plan de alfabetización académica tenga un impacto positivo en la calidad de los ensayos escritos por los alumnos de la muestra y el uso de sus conectores. En este sentido, para constatar si había correlación entre un uso adecuado de los marcadores discursivos en los textos y un buen desempeño en la producción de estos mismos, se aplicó el Test de Pearson ${ }^{3}$.

\subsection{Muestra}

Los informantes que proporcionaron la muestra fueron 40 estudiantes de primer año de la carrera de Pedagogía General Básica del campus Villarrica de la Pontificia Universidad Católica de Chile, quienes constituyen el total del universo de la muestra de estudiantes de primer aก̃o.

El corpus constó de 40 ensayos solicitados en un comienzo de la investigación y 40 ensayos al final de esta (8 meses después), los cuales fueron aplicados con el fin de medir la competencia comunicativa a nivel escrito de los estudiantes

3 Es una medida de la relación lineal entre dos variables aleatorias cuantitativas para medir su grado de correlación; en este caso, el uso adecuado de los enlaces en los ensayos y la calidad de estos últimos. 
que ingresan a la carrera, es decir, comprobar si son capaces de seguir una línea argumental clara y coherente, mediante el adecuado uso de sus marcadores discursivos y la planificación y revisión de sus textos.

\subsection{Instrumento}

Para explorar cómo operan los marcadores discursivos, no basta con determinar el significado de esta unidad lingüística, pues es necesario establecer las múltiples funciones que cumple en la progresión lógica del discurso de acuerdo con su contexto. Además, estos enlaces dependen de una variedad de factores que afectan su sentido y su papel dentro de los textos. Por lo tanto, ha sido necesario diseñar un instrumento de medición del uso y funcionamiento de los marcadores discursivos presentes en los exámenes que considera las siguientes dimensiones:

1. Evaluación inicial y final de cada ensayo mediante su triple revisión por evaluadores capacitados y calibrados, a través de una adaptación de la rúbrica para el Examen de Comunicación Escrita (tabla 1) elaborada por MIDE UC, cuya validación fue realizada previamente y su confiabilidad resultó ser alta. Este instrumento tiene como propósito medir la competencia comunicativa, a nivel escrito de los sujetos, es decir, comprobar si son capaces de seguir una línea argumental clara y coherente. Se estructura en una escala de 1 a 5, cuyo puntaje de corte para la reprobación es de 3 puntos, pues el texto no tendría una línea argumental clara.

2. Análisis de los tipos de marcadores discursivos presentes en los ensayos iniciales y finales.

3. Análisis de la yuxtaposición de los marcadores identificados con otros nexos.

4. Análisis de la adecuación de los marcadores identificados al tipo de género, a la situación de comunicación y a la clase de relación que quiere establecerse entre los enunciados o ideas.

\subsection{Dificultades en la identificación de los marcadores discursivos}

En general, surgieron algunas dificultades en la identificación de los marcadores discursivos en los ensayos, pues la mayoría de ellos no se presentaron entre pausas - que en los textos es-

\section{TABLA 1}

Dimensiones de la rúbrica de evaluación de los ensayos

\begin{tabular}{l|l}
\multicolumn{1}{c|}{ Dimensiones evaluadas } & \multicolumn{1}{c}{ Explicación } \\
1. Planificación del texto & $\begin{array}{l}\text { Grado de planificación del texto y consideración de su propósito, lectores, ideas, } \\
\text { estructura y fuentes. }\end{array}$ \\
\hline 2. Redacción 1 & $\begin{array}{l}\text { Grado de cohesión textual (correcto uso de la gramática oracional y de los conectores } \\
\text { que vinculan los enunciados. }\end{array}$ \\
\hline 3. Redacción 2 & $\begin{array}{l}\text { Grado en que el texto se estructura en párrafos y tiene una secuencia lógica de las } \\
\text { ideas. }\end{array}$ \\
\hline 4. Ordenamiento de ideas & $\begin{array}{l}\text { Grado en que ideas siguen una secuencia lógica, desarrolladas en cada uno de los } \\
\text { párrafos. }\end{array}$ \\
\hline 5. Tesis o idea central & $\begin{array}{l}\text { Grado en que el texto se identifica con un punto de vista sobre el tema enunciado y se } \\
\text { desarrolla en torno a este. }\end{array}$ \\
\hline 6. Argumentos & $\begin{array}{l}\text { Grado en que el texto formula argumentos válidos y coherentes para apoyar la tesis } \\
\text { ylos desarrolla. }\end{array}$ \\
\hline 7. Puntos de vista alternativos & $\begin{array}{l}\text { Grado en que el texto considera puntos de vista alternativos o contraargumentos a la } \\
\text { tesis para fortalecerla. }\end{array}$ \\
\hline 8. Estructura global & $\begin{array}{l}\text { Grado en que el texto presenta una introducción, un desarrollo y una conclusión } \\
\text { adecuados. }\end{array}$ \\
\hline
\end{tabular}


critos son representadas por comas - como un grupo entonacional aparte. Esto se puede deber a que probablemente el uso de los conectores no era consciente en la mayoría de los exámenes, ya que fueron escritos por alumnos universitarios de primer año, por tanto, su manejo de estos enlaces no era óptimo por no tener un mayor conocimiento sobre ellos. Además, debido a lo anterior, se presentaron dificultades para distinguir entre marcadores y conjunciones en los enunciados. Con el fin de distinguir los conectores de otras partículas, se constató que cumplieran con la mayoría de las propiedades gramaticales, mencionadas anteriormente, que permiten el reconocimiento de estos nexos, según Fuentes (1996) y Martín Zorraquino y Portolés (1999).

\subsection{Intervención}

La intervención consistió, como se mencionó anteriormente, en un plan de alfabetización académico de ocho meses de duración, aplicado a los alumnos y profesores de primer año de la carrera de Pedagogía General Básica. Por una parte, se asesoró semanalmente a los profesores en sus clases para desarrollar y evaluar adecuadamente géneros discursivos de su disciplina $y$, especialmente, el ensayo, como actividades epistémicas y de aprendizaje. Por otra parte, también se impartieron talleres dirigidos a los alumnos, donde se trabajó la escritura argumentativa, el ensayo, los momentos de la escritura y las habilidades de metacognición.

\section{Resultados}

\subsection{Frecuencias de las variables del instrumento de medición}

\subsubsection{Frecuencia de la evaluación global de los exámenes}

A partir de la evaluación global inicial de los ensayos que conforman el corpus, mediante la rúbrica para la revisión de ensayos, se obtuvieron los puntajes - en una escala de 1 a 5- de cada uno de los exámenes. Los resultados de la evaluación global inicial de los textos arrojaron que el promedio de los puntajes de los 40 ensa- yos analizados fue de 2,6 en una escala de 1 a 5 . De hecho, el puntaje que tuvo una mayor frecuencia de aparición en los textos fue 2,5, pues el 37,5\% de estos obtuvo esa calificación. Luego, los puntajes que le siguieron, en cuanto a frecuencia de aparición en los exámenes, fueron 3 y 2 con un $20 \%$ y un $14 \%$, respectivamente. De este modo, el 63,25\% de los alumnos reprobó el examen, pues tuvo un puntaje menor a 3 , por lo que se puede inferir que la mayoría de los estudiantes no tenía las competencias básicas para planificar y escribir ensayos adecuados.

Por el contrario, los resultados de la evaluación posterior al plan señalaron que el promedio de los puntajes de los 40 ensayos analizados fue de 3,2 en una escala de 1 a 5. De hecho, el puntaje que tuvo una mayor frecuencia de aparición en los textos fue 3, pues el $53 \%$ de los exámenes obtuvieron esa calificación. Luego, los puntajes que le siguieron, en cuanto a frecuencia de aparición en los exámenes, fueron 3,5 y 4 con un $23,1 \%$ y un $17,9 \%$, respectivamente. En consecuencia, es posible constatar que la totalidad de los alumnos aprobó el examen, pues obtuvieron puntajes iguales o superiores a 3, lo que en comparación con la evaluación inicial muestra que hubo un impacto del plan en estos alumnos y que se produjo un real avance de estos últimos. De hecho, son coherentes los resultados de la desviación estándar de las evaluaciones iniciales y finales de los ensayos, ya que fundamentan lo anteriormente enunciado, pues la desviación estándar de los primeros textos fue de 0.71 y la de los textos finales fue de 0.47 , lo que indica que posteriormente al plan la desviación estándar disminuyó y se aminoró la brecha entre los más altos puntajes y los más bajos, pues la mayoría de los estudiantes se ubicó en la media.

Con respecto a la evaluación de las diferentes dimensiones de la rúbrica, las menos logradas en la evaluación inicial fueron la planificación, el orden de las ideas y la contraargumentación, mientras que en la evaluación final fueron la redacción, la estructura y también los contraargumentos. Por el contrario, el aspecto 
con mayor desempeño en la evaluación inicial fue la tesis y en la evaluación final continuó siendo la tesis y también la presencia de argumentos y la planificación. La evaluación de esta última dimensión nos indica que posiblemente los elevados resultados en los ensayos finales se deben al dominio de las habilidades de planificación y revisión de textos y, por tanto, al fortalecimiento de las competencias metacognitivas para autorregular los procesos de escritura, pues fueron estos los aspectos desarrollados principalmente en la intervención. También, es posible observar que en ambas evaluaciones la contraargumentación continuó siendo una habilidad poco desarrollada; esto evidentemente se debe a que es una de las más complejas y extensas de trabajar, lo que se reflejó en el uso inadecuado de los marcadores de oposición, que en varias ocasiones, especialmente en la evaluación inicial, se utilizaron no solo para marcar relaciones de oposición, sino para otro tipo de relaciones como de causaefecto o de adición. De hecho, si bien los marcadores más frecuentes en ambos corpus fueron los de oposición, las relaciones que realmente indicaban algunos de ellos no eran de este tipo. Asimismo, uno de los aspectos que más se fortaleció luego de la intervención fue la presencia de argumentos, y esto se manifestó en una utilización frecuente y correcta de marcadores de adición y continuidad, y si bien los enlaces de causa-efecto se utilizaron con menor frecuencia, sí mostraron un adecuado uso, más que otros nexos. Estos resultados se presentan en la tabla 2.

\subsubsection{Frecuencia de tipos de marcadores discursivos}

Se analizó la frecuencia de aparición de cada tipo de marcador discursivo en los textos de ambas muestras: Ia inicial y la posterior a la intervención. Los datos arrojaron — como puede observarse en el gráfico 1 - que el tipo de conector que más se presentó en los textos de ambos corpus fue el de oposición. Luego le siguieron los enlaces de adición y de causalidad. Por el contrario, los nexos que se encontraron en menor cantidad al interior del corpus fueron los de equivalencia y orden.

Los enlaces más frecuentes en la muestra fueron "pero", "por ejemplo", "también”, "además" y "asi", dentro de los cuales no hay de causalidad.

En cuanto a las funciones que cumplieron este tipo de marcadores en los textos argumentativos, fueron principalmente la de oponerse a la idea anterior para defender los propios argumentos a través del conector "pero" y la de añadir ideas y ejemplos concretos, lo que se demuestra en la abundante utilización de los enlaces “por ejemplo”, “también”, “además” y “así". De este modo, es posible observar que unas de las operaciones lógicas fundamentales en el discurso argumentativo, como son la causa y el efecto y la contraargumentación (Anscombre y Ducrot, 1994; Anscombre, 1995), se presentan escasamente.

\section{TABLA 2}

Cuadro comparativo de los aspectos evaluados en los ensayos iniciales y finales

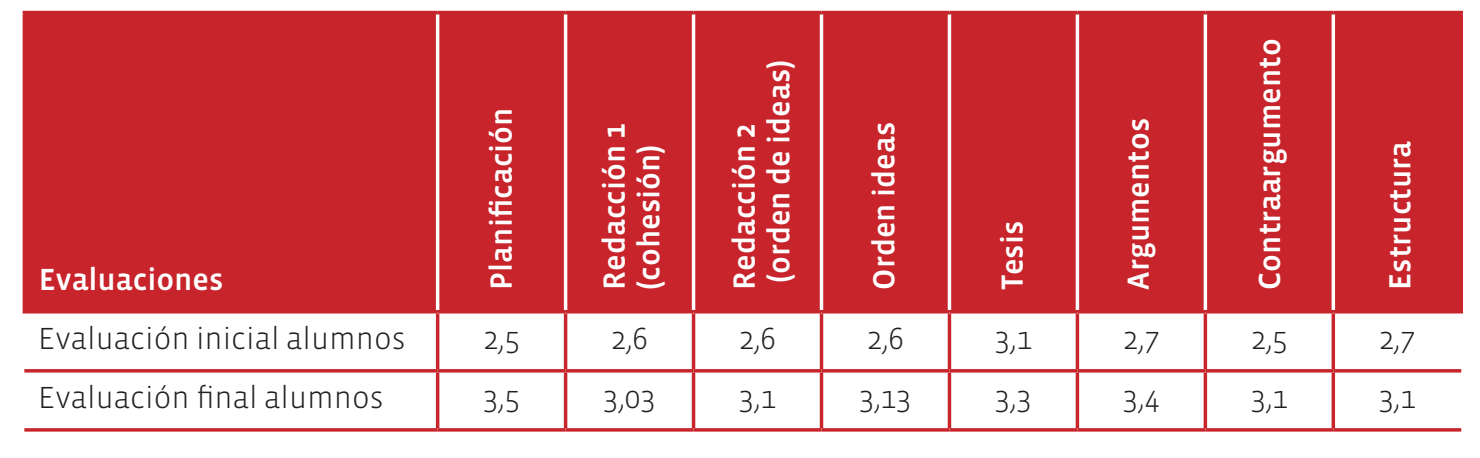




\section{GRÁFICO 1}

Frecuencia de aparición de los tipos de marcadores discursivos en el corpus

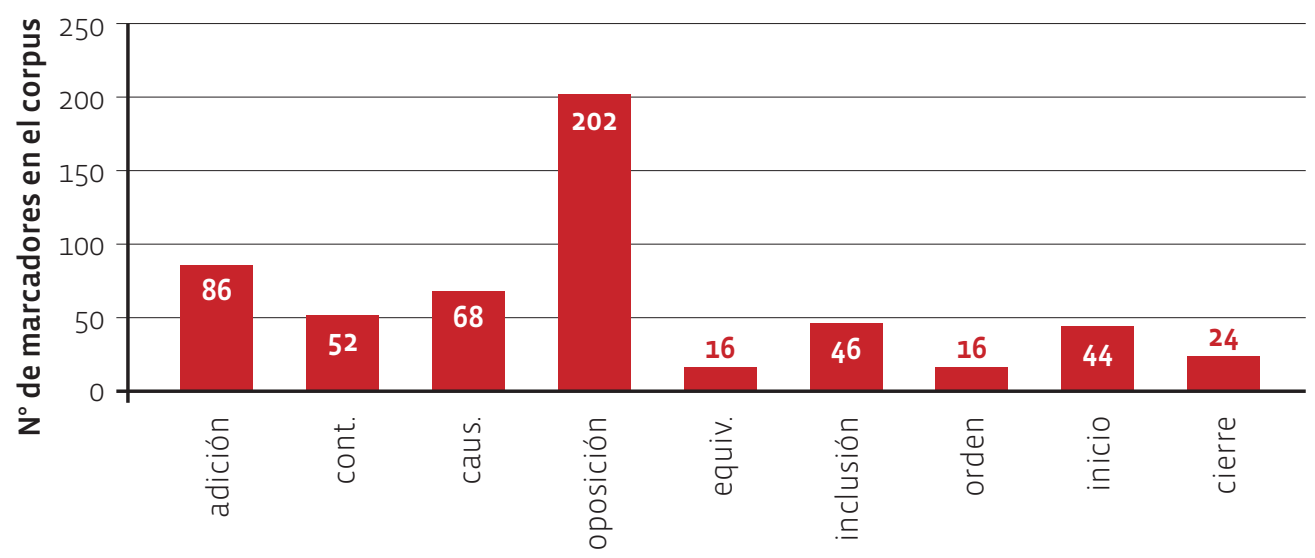

Tipos de marcadores discursivos

\subsubsection{Frecuencia de yuxtaposición en los marcadores discursivos}

A partir del análisis de la totalidad de los marcadores identificados en el corpus inicial, fue posible constatar que solo un $5 \%$ del total de los conectores encontrados en los ensayos de la muestra funcionaron en combinación con otros nexos, mientras que en el corpus posterior al plan se encontraron solo un $3 \%$ de enlaces yuxtapuestos.

Cabe señalar que, en general, los marcadores yuxtapuestos no se utilizaron de modo adecuado, debido a informalidad o redundancia. Esto indica - como se constató también en los ensayos iniciales - que probablemente la yuxtaposición de los conectores provoca problemas, ya que no se utiliza de manera debida y no se adapta a las inferencias lógicas, al tipo de discurso y a la situación de comunicación.

A continuación se presenta un ejemplo de yuxtaposición

(1) Ejemplo: Pero toda mujer, aún en silencio, conoce su rol, reconoce su poder, la mujer fue creada según cuenta el génesis como necesidad de acompañaral hombre, y no de servirlo. Así entonces, el que hoy se busque y luche por la reivindicación de la mujer es una batalla principalmente por la dignidad de esta, por el reconocimiento, pues toda sociedad evoluciona por lo que nuestros comportamientos y relaciones, también deben hacerlo.

\subsubsection{Frecuencia de adecuación de los marcadores discursivos}

La mayoría de los conectores identificados en los textos resultaron ser pertinentes, es decir, fueron usados de un modo adecuado y su funcionamiento se adaptó al tipo de discurso argumentativo, a su situación de enunciación y al tipo de relación que pretendía establecer.

De este modo, en el corpus inicial se obtuvo un promedio de 6,3 marcadores pertinentes por examen, mientras que en el corpus final el promedio fue de 7 marcadores adecuados por ensayo.

Así, es posible constatar que un 91\% de los marcadores de los exámenes iniciales tuvo un uso pertinente, mientras que un $98 \%$ de los enlaces encontrados en la muestra luego de la intervención fue adecuado.

Estos resultados también comprueban que hubo una real mejoría en la calidad del uso de los marcadores presentes en los textos luego de la intervención, pues en los primeros resultados hubo un $9 \%$ de enlaces inadecuados, cifra que disminuyó considerablemente a un 2\%. 
A continuación se presentan dos ejemplos de enlaces de oposición utilizados inadecuadamente.

(2) Ejemplo: Se escandalizan ante la informalidad del correo electrónico y creen que esto lleva a una comunicación menos profunda, pero entender que es un mensaje rápido y no comunica cosas muy importantes.

(3) Ejemplo: El tiempo avanza, y junto con él avanzan también las exigencias y la efectividad en la vida, en el trabajo, en el estudio. Sin embargo, también avanza la tecnología.

Es posible observar en los dos ejemplos anteriores que se presentan marcadores de oposición; sin embargo, no hay una relación lógica de oposición entre las ideas presentadas, sino que se establecen relaciones de adición y consecuencia. Por lo tanto, el uso de estos enlaces sería no pertinente, pues no representan las relaciones lógicas que realmente existen en los enunciados

Con respecto a la cantidad de marcadores discursivos pertinentes encontrados en los ensayos, en relación con los puntajes de los textos en que estaban insertos, es posible aseverar que los textos con puntajes mayores (3,5 y 4) presentaron una mayor cantidad de marcadores discursivos pertinentes que los textos con puntaje más bajo, lo que demuestra que los ensayos con un puntaje más alto ostentaron una mayor frecuencia de conectores y un funcionamiento más adecuado. Con respecto a la relación de los marcadores discursivos no adecuados con los puntajes de los exámenes en que estaban insertos, los textos que presentaron una mayor cantidad de conectores no pertinentes fueron aquellos que obtuvieron nota 1,5. Cabe señalar que los exámenes con nota 3,5 y 4 presentaron solo un conector no adecuado, lo que comprueba que los textos con un puntaje más alto en la evaluación ostentaron un uso más apropiado de los enlaces.

Ahora bien, si obtenemos el promedio de conectores pertinentes y no pertinentes, en re- lación con la cantidad de textos presentes por cada uno de los puntajes obtenidos en los exámenes, es posible observar que la cantidad de enlaces adecuados utilizados es directamente proporcional al puntaje del texto y, por tanto, a su calidad. Es decir, en este caso se infiere que es probable que a mayor uso de marcadores adecuados, mayor coherencia de su línea argumental.

Para comprobar el nivel de la correlación entre un mayor uso de marcadores pertinentes y una mayor calidad de los textos, se aplicó a estos resultados el Test de Pearson (fórmula Coeficiente R2) y se obtuvo lo siguiente (tabla 3):

\section{TABLA 3}

Correlación Pearson entre la adecuación de los marcadores y la coherencia de los textos

\begin{tabular}{c|c|c}
$\begin{array}{c}\text { Puntajes } \\
\text { de textos }\end{array}$ & $\begin{array}{c}\text { Promedio del } \mathbf{n}^{\circ} \text { de } \\
\text { marcadores adecuados en } \\
\text { relación con la cantidad } \\
\text { de textos por puntaje }\end{array}$ & Pearson \\
\hline 1 & 2 & $\mathbf{0 , 9 5 3 5 5 9 4 5}$ \\
\hline 1,5 & 4,8 & \\
\hline 2 & 5,2 & \\
\hline 2,5 & 5,6 & \\
\hline 3 & 7,25 & \\
\hline 3,5 & 9,5 &
\end{tabular}

En consecuencia, es posible comprobar, a partir de los resultados obtenidos, que efectivamente sí existe una correlación entre ambas variables: la adecuación de los marcadores y la calidad de los textos, pues las cifra arrojada, 0,95, es significativa.

\section{Discusión y conclusiones}

Este estudio ha constatado la importancia que tienen los marcadores discursivos para la estructuración de los discursos. Estos elementos no solo organizan y ordenan los componentes de la argumentación, sino que también son capaces 
de guiar las inferencias lógico-semánticas de un texto, orientar los razonamientos y establecer relaciones de causalidad, adición, continuidad, oposición y cierre, entre otras (Anscombre y Ducrot, 1994; Lo Cascio, 1998; Portolés, 1998). De hecho, estos enlaces son capaces de contribuir a la coherencia, a la cohesión y a la progresión lógica del texto, propiciando así su desarrollo semántico-estructural y su efectividad comunicativa. Por lo tanto, la claridad de la línea argumental e inferencial de los textos puede ser dirigida por los marcadores, los cuales facilitan la tarea del lector y del interlocutor en la codificación y decodificación del discurso y en la comprensión de este.

Además, los marcadores pueden fijar claramente las fases del esquema argumentativo. Por ejemplo, en el marco inicial pueden introducir el tema, el propósito del texto o la tesis; en el desarrollo del tema pueden añadir datos e ideas y fijar relaciones de oposición y de causa-consecuencia, lo que permite ingresar argumentos y contraargumentos; y en el marco final pueden establecer conclusiones, recapitular las ideas, presentar proyecciones, confirmar la tesis e instaurar el cierre del texto.

En efecto, los resultados obtenidos demostraron que el nivel de eficacia en el uso de los marcadores discursivos es un excelente indicador de la calidad de un texto; de hecho, los textos con puntajes más altos también demostraron tener una mayor utilización de enlaces y una mayor adecuación de estos. También se constató que la aplicación de un plan de literacidad académica tuvo un impacto beneficioso, ya que los estudiantes mejoraron los puntajes de sus ensayos y también la utilización de sus conectores, tanto así que en la evaluación diagnóstica se detectó un 9\% de marcadores inadecuados y en la postevaluación luego del plan se encontró solo un $2 \%$ de enlaces inadecuados, es decir, el correcto uso de estos nexos mejoró en un $7 \%$. Asimismo, los ensayos anteriores al plan tuvieron un puntaje promedio de 2,6, bajo el estándar mínimo de 3 puntos, y luego de este tuvieron un puntaje promedio de 3,2, es decir, todos los estudiantes aprobaron el examen.

Con respecto a las conclusiones específicas que se desprenden del análisis de los marcadores discursivos identificados en el corpus, se constató lo siguiente.

En primer lugar, el uso adecuado de los marcadores resultó ser directamente proporcional al nivel de coherencia y claridad de los ensayos, como se señaló anteriormente, pues los textos con evaluaciones más altas presentaron conectores con un funcionamiento más lógico y adecuado al contexto. Asimismo, los exámenes con evaluaciones más bajas mostraron un uso escaso o inadecuado de los enlaces, lo que afectó la claridad de su línea argumental. Posiblemente, los marcadores contribuyeron efectiva y concretamente al procesamiento de la información en los textos y a la orientación lógica, semántica e inferencial de las ideas y argumentos, lo que se demuestra en el corpus posterior a la intervención, que ciertamente presenta puntajes más altos y menos conectores utilizados inadecuadamente. En este sentido, las cifras son elocuentes, pues los estudiantes pasaron de tener 2,6 puntos en sus ensayos a tener 3,2 puntos y el porcentaje de marcadores inadecuados presentes en sus textos pasó de ser un $9 \%$ a ser un $2 \%$.

En segundo lugar, se descubrió un uso excesivo y muchas veces inadecuado de los marcadores de oposición y especialmente de "pero"; de hecho, esta clase de enlace fue la más frecuente en el corpus (del 20\% al 26\%), seguida por la de adición (“también” y "además"), la de causalidad ("así") y la de continuidad, respectivamente. En varias ocasiones el uso de estos tipos de conectores no concordaba con la relación lógica que se pretendía establecer entre los enunciados, pues se marcaba oposición en segmentos que claramente no se oponían, sino que se añadían, por ejemplo. Como consecuencia de esto es posible inferir que la fuerza argumentativa de los textos se ve debilitada al presentar una mayoría 
de enlaces de oposición, pues son modificadores desrealizantes: poseen un lugar descendente en las escalas argumentativas fijadas por los topoi y no siguen la misma dirección argumentativa (Anscombre y Ducrot, 1994; cfr. Escandell, 1993: 123-125). Por ende, se debilitan los argumentos enunciados y, así, los ensayos presentan una menor calidad, lo que se refleja en sus bajos puntajes. No obstante, luego del plan este tipo de enlaces mostró un uso más adecuado, ya que al comienzo se presentó un $16 \%$ inadecuado y al final un $4 \%$, lo que demuestra el efecto positivo de esta iniciativa.

En tercer lugar, llama la atención el uso escaso que tuvieron los marcadores de causalidad (12\% en ambas muestras), los cuales resultan fundamentales para la orientación de las inferencias en los textos argumentativos. Su valiosa contribución como modificadores realizantes, pues apoyan la línea argumental del texto y le confieren fuerza argumentativa (Anscombre y Ducrot, 1994; cfr. Escandell, 1993: 123-125), no fue aprovechada en los ensayos, lo que se tradujo en una modesta presencia de relaciones inferenciales y lógicas de causa-efecto y en una falta de habilidades de razonamiento lógico. Probablemente esta insuficiencia se manifestó en los bajos puntajes que tuvieron los textos en la muestra diagnóstica, ya que en la muestra final, si bien estos nexos tuvieron una baja frecuencia, presentaron un uso adecuado, a diferencia del corpus inicial que mostró un 3\% de estos enlaces usados inadecuadamente.

En cuarto lugar, se constató que varias de las yuxtaposiciones que presentaron algunos de los conectores produjeron la inadecuación de estos al discurso, pues se relacionaron nexos que se contradecían entre sí o cuya vinculación no tenía sentido. Por otra parte, la frecuencia de yuxtaposición disminuyó en un $2 \%$ en el corpus final, lo que se relaciona con el mayor nivel de adecuación en la utilización de sus marcadores discursivos y en la mayor calidad de sus textos, lo que nuevamente comprueba que el plan tuvo efectos beneficiosos en el nivel de producción escrita de los estudiantes.

Con respecto a las implicaciones pedagógicas de esta investigación, es necesario destacar que la efectividad e impacto positivo que tuvo la aplicación del programa de alfabetización académica se debió, por una parte, a que participaron la mayoría de los profesores de diversas áreas del currículo del programa de formación inicial docente; por lo tanto, los estudiantes tuvieron la oportunidad de desarrollar la escritura académica de modo sistemático en varias disciplinas. Por otra parte, a que también se enfocó en asesorar a los profesores en el desarrollo de esta competencia en sus alumnos y en sus áreas específicas, como una herramienta de aprendizaje y construcción de conocimientos (Bazerman y otros, 2005; Carlino, 2005 y 2013). De este modo, los docentes fueron capaces de monitorear los procesos de escritura: planificación, escritura y revisión; orientarlos; evaluarlos continuamente, e introducir a sus estudiantes en la escritura académica y el género ensayo. Esto se puede constatar en los altos puntajes de la dimensión de planificación en la evaluación de los ensayos posteriores al plan, lo que seguramente se debe al mayor dominio de las habilidades de planificación y revisión de textos por parte de los estudiantes y, por tanto, al fortalecimiento de sus competencias metacognitivas para autorregular los procesos de producción escrita (Cassany, 2000; Didactext, 2003; Flower y Hayes, 1981; Grabe y Kaplan, 1996).

En este sentido, el uso de los marcadores discursivos se presenta como una estrategia clave para medir la producción de los estudiantes, pues estas unidades permiten el desarrollo de un propósito argumentativo claro y coherente. Tener conocimientos sobre esto proporciona mayor base empírica para diseñar intervenciones pedagógicas remediales en un área de gran importancia para el desarrollo del discurso académico estudiantil, especialmente, porque en la última década los alumnos de educación 
superior presentan dificultades de redacción para adaptarse a la situación de comunicación académica, debido a la interferencia de la oralidad (Errázuriz, 2010). Así, es posible inferir que, ciertamente, los jóvenes están cada vez menos expuestos a otros contextos de enunciación que no sean coloquiales y familiares dentro del registro oral, lo que - junto a los medios de comunicación masiva y los avances tecnológicos- está profundizando el problema (Errázuriz, 2010: 203, y Tusón, 1991: 18-19). Todo esto, sumado al creciente desarrollo de la tecnología y multimedia con herramientas como el chat, genera códigos escritos alternativos y de uso coloquial. De hecho, los marcadores discursivos más utilizados en el corpus son enlaces más bien ligados a contextos coloquiales y orales que a situaciones propias de textos académicos, por ejemplo, el conector "pero" (Errázuriz, 2010).

Por otra parte, según diversas investigaciones (Bazerman y otros, 2005; Carlino, 2004, 2005, 2009 y 2013; Castelló, 2009; Errázuriz y Fuentes, 2010, y Errázuriz y Fuentes, 2012), los docentes de programas de formación de profesores, en general, no apoyan ni supervisan lo suficiente los procesos de análisis y producción de textos en sus clases; por lo tanto, es urgente que lo comiencen a hacer más. Para esto deben dar la oportunidad de leer y escribir textos auténticos en clases y de realizar un seguimiento de estas tareas y de las etapas de producción (Carlino, 2005; Didactext, 2003): planificación, redacción y revisión. Mediante la guía y modelamiento del profesor, el alumno entenderá los parámetros que debe seguir para la escritura en su disciplina y el manejo de los conectores. También es importante que el docente comunique de modo claro cuáles serán los criterios que evaluará en las tareas de producción escrita, además de precisar la situación de comunicación del texto a escribir, la estructura y el propósito de este.

Finalmente, en relación con las proyecciones que posee esta investigación, podría extenderse y aplicarse a un corpus de estudiantes de programas de formación inicial docente de mayor tamaño de diversas universidades chilenas, cuyos resultados y conclusiones fueran generalizables y predictivos de sus competencias comunicacionales y de sus falencias discursivas, para así contar con conocimientos que contribuyan a mejorar la calidad de los programas de formación de profesores y la educación escolar chilena.

\section{Bibliografía citada}

Ahumada, Pedro, 2005: "La evaluación auténtica: un sistema para la obtención de evidencias y vivencias de los aprendizajes", Perspectiva Educacional 45, primer semestre, 11-24.

Álvarez, Teodoro y Roberto Ramírez, 2006: "Teorías o modelos de producción de textos en la enseñanza y el aprendizaje de la escritura", Didáctica (Lengua y Literatura), vol. 18, 26-60.

Anscombre, Jean, 1995: "Semántica y léxico: topoï, estereotipos y frases genéricas”, Revista Española de Lingüística 25, 2, 297-310.

Anscombre, Jean y Oswald Ducrot, 1994: La argumentación en la lengua, Madrid: Gredos.

BAJTIN, Mijail, 1996: Estética de la creación verbal, México: Siglo XXI.

Bazerman, Charles, Joseph Little, Lisa Bethel, Teri Chavkin, Danielle Fouquette y Janet Garufis, 2005: Reference Guide to Writing Across the CurricuIum, West Lafayette: Parolor Press.

Beaugrande, Robert y Wolfgang Dressler, 1997: Introducción a la lingüística del texto, Barcelona: Ariel.

Bereiter, Carl y Marlene Scardamalia, 1987: The Psychology of Written Composition, New Jersey: LEA.

Blakemore, Dianne, 2002: Relevance and linguistic meaning: the semantics and pragmatics of dis- 
course markers, Cambridge: Cambridge University Press.

BrockRIEDE, Wayne, 1993: “¿Dónde hay un argumento?”, comunicación presentada en el Seminario Internacional: Lógica Informal y Teoría de la Argumentación. Santiago, Chile.

Bronckart, Jean Paul, 2010: Desarrollo del lenguaje y didáctica de las lenguas, Buenos Aires: Miño y Dávila.

Calsamiglia, Helena y Amparo Tusón, 2001: Las cosas del decir, Barcelona: Ariel.

Camps, Ana, 2003: "Miradas diversas a la enseñanza y el aprendizaje de la composición escrita", Lectura y Vida, año 24, 4, 2-11.

Candlin, Christopher y Ken Hyland (eds.), 1999: Writing: text, processes and practices, New York: Longman.

Carlino, Paula y Constanza Padilla, 2010: "Alfabetización académica e investigación-acción: Enseñar a elaborar ponencias en la clase universitaria” en Giovanni PARodi (ed.): Alfabetización académica y profesional en el siglo XXI: Leer y escribir desde las disciplinas, Santiago: Planeta, 153-182.

Carlino, Paula, 2013: "Alfabetización Académica diez años después", Revista Mexicana de Investigación, vol. 18, núm. 57, 355-381.

Carlino, Paula, 2009: "Desarrollo profesional de docentes para leer y escribir a través del currículum" en Paula Carlino y Silvia Martínez (coords.): La lectura y la escritura, un problema asunto de todos, Neuquén: Universidad Nacional de Comahue.

CARLINo, Paula, 2005: Escribir, leer y aprender en la universidad. Una introducción a la alfabetización académica, Buenos Aires: Fondo de Cultura Económica.

Carlino, Paula; 2004: "Escribir a través del currículum: tres modelos para hacerlo en la universidad”, Lectura y Vida. Revista Latinoamericana de Lectura, año 25, nº 1, 16-27.
Cassany, Daniel, 2000: Construir la escritura, BarceIona: Paidós

Castelló, Montserrat (coord.), 2009: Escribir y comunicarse en contextos científicos y académicos, Barcelona: Graó.

Castro, María Cristina, Laura Aurora Hernández y Martín SÁnchez, 2010: "El ensayo como género académico: Una aproximación a las prácticas de escrituras en la universidad pública mexicana" en Giovanni PARodı (ed.): Alfabetización académica y profesional en el siglo XXI: Leer y escribir desde las disciplinas, Santiago: Ariel.

Condemarín, Mabel y Alejandra Medina, 2000: EvaIuación auténtica de los aprendizajes. Un medio para mejorar las competencias en lenguaje y comunicación, Santiago: Andrés Bello.

Cueva, Alberto, 2008: "Estado de la cuestión sobre las características gramaticales de los marcadores discursivos del español", Forma y Función 21, 87-106.

DidacteXt (grupo), 2003: "Modelo sociocognitivo, pragmalingüístico y didáctico para la producción de textos escritos", Didáctica (Lengua y Literatura), vol. 15, 77-104.

Ducrot, Oswald, 2001: El decir y lo dicho: polifonía de la enunciación, Barcelona: Paidós.

Errázuriz, María Constanza, 2012: "Análisis del uso de los marcadores discursivos en argumentaciones escritas por estudiantes universitarios", Revista Perfiles Educativos, volumen XXXIV, $n^{\circ}$ 136, 98-117.

Errázuriz, María Constanza, 2010: "Marcas de oralidad en textos académicos estudiantiles", Revista de Humanidades 21, 183-205.

Errazuriz, María Constanza y Liliana Fuentes, 2012: "Diseño, implementación y evaluación de una propuesta de intervención en Alfabetización Académica en primer año de Pedagogía General Básica en la Sede Villarrica de la UC”, Revista Onomázein 25, 287-313. 
Errazuriz, María Constanza y Liliana Fuentes, 2010: "Evaluación y desarrollo de una propuesta centrada en la producción de textos en estudiantes de primer año de pedagogía en educación general básica”, Revista Sembrando Ideas 4, 39-53.

Errázuriz, María Constanza y María Verónica SánCHez (enviado): "El desarrollo de la escritura académica en la formación inicial docente: El centro de escritura como estrategia de modelamiento de tutores y estudiantes", Revista Universitas Psycological.

Escandell, Victoria, 1993: Introducción a la pragmática, Barcelona: Anthropos.

Ferreiro, Emilia, 2008: Pasado y presente de los verbos leer y escribir, Buenos Aires: Fondo de Cultura Económica.

Flower, Linda y John HAYES, 1981: "A cognitive process theory of writing", College Composition and Communication 31, 132-149.

Flower, Linda, 1989: "Cognition, context and theory building", College Composition and Comunication 40, 3, 122-142.

Foster, David y David Russell, 2002: Writing and Learning in Cross-National Perspective, Urbana: National Council of Teachers of English y Mahwah: Lawrence Erlbaum Associates, Inc.

Fuentes, Claudia, 1996: La sintaxis de los relacionantes supraoracionales, Madrid: Arco libros.

García Parejo, Isabel, 2011: "Exposición escrita, escuela y sociedad" en Isabel García Parejo (coord.): Escribir textos expositivos en el aula, Barcelona: GRAÓ.

Grabe, William y Robert KaPLAN, 1996: Theory and Practice of Writing, New York: Longman.

Grice, Paul, 1975: “Lógica y conversación” en Luis Manuel Valdés Villanueva (ed.), 2005: La búsqueda del significado: lecturas de filosofía del lenguaje, Madrid: Tecnos.
Hyland, Ken, 2004: Disciplinary Discourse. Social interactions in academic writing, London: Longman.

Lo Cascio, Vincenzo, 1998: Gramática de la argumentación, Madrid: Alianza Universidad.

Loureda, Óscar y Esperanza Acin (eds.), 2010: Los estudios sobre marcadores del discurso en español, hoy, Madrid: Arco Libros.

Martín Zorraquino, María Antonia y José Portolés, 1999: "Los marcadores del discurso" en Ignacio Bosque y Violeta Demonte (coords.): Gramática descriptiva de la lengua española, vol. 3, Madrid: Espasa-Calpe.

Martínez, María Cristina, 2001: Análisis del discurso y práctica pedagógica, Santa Fe: Homo Sapiens.

MeCKES, Lorena, 2011: Formación inicial docente, CEPPE, Pontificia Universidad Católica.

Ministerio de Educación, 2005: Informe Comisión Formación Inicial Docente, Santiago: Serie Bicentenario.

MiRas, Mariana e Isabel Solé, 2009: "La elaboración del conocimiento científico y académico" en Montserrat Casteloo (coord.): Escribir y comunicarse en contextos científicos y académicos, Barcelona: Graó, 83-112.

Montolío, Estrella, 2001: Conectores de la lengua escrita, Barcelona: Ariel.

Nystrand, Martin, 1989: "A social-interactive model of writing", Written Communication, vol. 6, 1, 6-85.

Oliva, Iván, Nicolás Díaz, Paulina Larrosa, Paulo Contreras y Christian Miranda, 2010: "Dimensiones de fractura cognitiva en formación inicial docente en Chile: Un estudio de casos en tres contextos formativos", Estudios Pedagógicos XXXVI, $n^{\circ} 1$, 177-189. 
Orealc/Unesco, Llece y Ana Atorresi, 2010: Escritura. Un estudio de las habilidades de los estudiantes de América Latina y el Caribe, Santiago: Productora Gráfica Andros Ltda.

Parodi, Giovanni, 2012: “¿Qué se lee en los estudios doctorales?: Estudio empírico basado en géneros a través del discurso académico de seis disciplinas", RLA. Revista de Lingüística Teórica y Aplicada 50 (2), 89-119.

Parodi, Giovanni (ed.), 2010: Alfabetización académica y profesional en el siglo XXI: Leer y escribir desde las disciplinas, Santiago: Ariel.

Parodi, Giovanni, Marianne Peronard y Romualdo Ibẫez, 2010: Saber leer, Buenos Aires: Aguilar.

Perelman, Chaim y Lucie Olbrechts Tyteca, 2009: Tratado de la argumentación: la nueva retórica, Madrid: Gredos.

Pérez, Mónica y Olly Vega, 2001: Claves para la conexión textual, Santiago: Ediciones UC.

Pons, Salvador, 1998: Conexión y conectores. Estudio de su relación en el registro informal de la lengua, Valencia: Universitat de València.

Portolés, José, 2010: "Los marcadores y la estructura informativa” en Óscar LouReda y Esperanza Acin (eds.): Los estudios sobre marcadores del discurso en español, hoy, Madrid: Arco Libros, 281325.

Portolés, José, 1998: Marcadores del discurso, Barcelona: Ariel.

Pottier, Bernard, 1993: Semántica General, Madrid: Gredos.

Ruız, Maite, 2009: Evaluación de lengua escrita y dependencia de lo literal, Barcelona: Graó.

Sabaj, Omar y Denisse LandeA, 2012: "Descripción de las formas de justificación de los objetivos en artículos de investigación en español de seis áreas científicas”, Revista Onomázein 25, 315-344.

Silva-Corvalán, Carmen, 2001: "Análisis del Discurso" en Sociolingüística y pragmática del español,
Washington, D.C.: Georgetown University Press.

Slotte, Virpi y Kirsti LonKA, 2001: "Note taking and essay writing” en Päivi Tynjala, Lucia Mason y Kirsti Lonka (eds.): Writing as a Learning Tool. Integrating Theory and Practice, Dordrecht: Kluwer Academic Publishers.

SPERBER, Dan y Deirdre WiLson, 1994: La relevancia: comunicación y procesos cognitivos, Madrid: Visor.

Swales John y Christine Feak, 2004: Academic Writing for Graduate Students, Michigan: The University of Michigan Press.

Teberosky, Ana, 2009: "El texto académico" en Montserrat CASTELló (coord.): Escribir y comunicarse en contextos científicos y académicos, Barcelona: Graó, 17-46.

Toulmin, Stephen, 1993: “La estructura de los argumentos", comunicación presentada en el Seminario Internacional: Lógica Informal y Teoría de la Argumentación. Santiago, Chile.

Toulmin, Stephen, 1958: The uses of argument, Cambridge: Cambridge University Press.

Tragant, Elsa, 1996: "La corrección de la expresión escrita: las preferencias de los estudiantes”, Didáctica 8, 319-332.

Tusón, Amparo, 1991: "Las marcas de la oralidad en la escritura", Signos. Teoría y práctica de la educación 12, 14-19.

Van Dıjk, Teun, 1997: La ciencia del texto, Barcelona: Paidós.

Van Eemeren, Frans y Rob Grootendorst, 2002: Argumentación, Comunicación y Falacias, Santiago: Ediciones Universidad Católica.

VIGnaux, George, 1986: "El universo sistemático de los argumentos" en La argumentación. Ensayo de la lógica discursiva, Buenos Aires: Hachette.

Weinberg, Liliana, 2007: Pensar el ensayo, México: Siglo XXI. 
Wenzel, John, 1993: "Perspectivas sobre el argumento", comunicación presentada en el Seminario Internacional: Lógica Informal y Teoría de la Argumentación. Santiago, Chile.

Weston, Anthony, 1994: Las claves de la argumentación, Barcelona: Ariel. 